\title{
OPEN Accuracy of high-frequency oscillations recorded intraoperatively for classification of epileptogenic regions
}

\author{
Shennan A. Weiss ${ }^{1,2,3}$, Richard J. Staba ${ }^{6}$, Ashwini Sharan ${ }^{11}$, Chengyuan Wu ${ }^{11}$, \\ Daniel Rubinstein ${ }^{10}$, Sandhitsu Das ${ }^{12}$, Zachary Waldman $^{10}$, Iren Orosz ${ }^{6}$, Gregory Worrell ${ }^{4,5}$, \\ Jerome Engel Jr $\mathrm{r}^{6,7,8,9}$ \& Michael R. Sperling ${ }^{10 \bowtie}$
}

To see whether acute intraoperative recordings using stereo EEG (SEEG) electrodes can replace prolonged interictal intracranial EEG (iEEG) recording, making the process more efficient and safer, 10 min of iEEG were recorded following electrode implantation in 16 anesthetized patients, and 1-2 days later during non-rapid eye movement (REM) sleep. Ripples on oscillations (RonO, $80-250 \mathrm{~Hz}$ ), ripples on spikes (RonS), sharp-spikes, fast RonO (fRonO, 250-600 Hz), and fast RonS (fRonS) were semi-automatically detected. HFO power and frequency were compared between the conditions using a generalized linear mixed-effects model. HFO rates were compared using a two-way repeated measures ANOVA with anesthesia type and $\mathrm{SOZ}$ as factors. A receiver-operating characteristic (ROC) curve analysis quantified seizure onset zone ( $\mathrm{SOZ}$ ) classification accuracy, and the scalar product was used to assess spatial reliability. Resection of contacts with the highest rate of events was compared with outcome. During sleep, all HFOs, except fRonO, were larger in amplitude compared to intraoperatively $(p<0.01)$. HFO frequency was also affected $(p<0.01)$. Anesthesia selection affected HFO and sharp-spike rates. In both conditions combined, sharp-spikes and all HFO subtypes were increased in the SOZ $(p<0.01)$. However, the increases were larger during the sleep recordings $(p<0.05)$. The area under the ROC curves for $\mathrm{SOZ}$ classification were significantly smaller for intraoperative sharp-spikes, fRonO, and fRonS rates $(p<0.05)$. HFOs and spikes were only significantly spatially reliable for a subset of the patients $(p<0.05)$. A failure to resect fRonO areas in the sleep recordings trended the most sensitive and accurate for predicting failure. In summary, HFO morphology is altered by anesthesia. Intraoperative SEEG recordings exhibit increased rates of HFOs in the SOZ, but their spatial distribution can differ from sleep recordings. Recording these biomarkers during non-REM sleep offers a more accurate delineation of the $\mathrm{SOZ}$ and possibly the epileptogenic zone.

For patients with medically refractory epilepsy who are candidates for epilepsy surgery, invasive intracranial EEG (iEEG) monitoring is often required. Intraoperative electrocorticography (ECoG) using subdural electrodes ${ }^{1}$, and occasionally intrahippocampal depth electrodes ${ }^{2,3}$, are often used in place of prolonged iEEG recordings in childrenn $n^{4,5}$, and in adults with a clear pathological substrate ${ }^{1,6,7}$. It is unclear if acute recordings from stereo

\footnotetext{
${ }^{1}$ Department of Neurology, State University of New York Downstate, Brooklyn, NY 11203, USA. ${ }^{2}$ Department of Physiology and Pharmacology, State University of New York Downstate, Brooklyn, NY 11203, USA. ${ }^{3}$ Department of Neurology, New York City Health + Hospitals/Kings County, Brooklyn, NY, USA. " Department of Neurology, Mayo Systems Electrophysiology Laboratory (MSEL), Rochester, USA. ${ }^{5}$ Department of Physiology and Biomedical Engineering, Mayo Clinic, Rochester, MN 55905, USA. ${ }^{6}$ Department of Neurology, David Geffen School of Medicine at UCLA, Los Angeles, CA 90095, USA. 'Department of Neurobiology, David Geffen School of Medicine at UCLA, Los Angeles, CA 90095, USA. ${ }^{8}$ Department of Psychiatry and Biobehavioral Sciences, David Geffen School of Medicine at UCLA, Los Angeles, CA 90095, USA. ${ }^{9}$ Brain Research Institute, David Geffen School of Medicine at UCLA, Los Angeles, CA 90095, USA. ${ }^{10}$ Department of Neurology and Neuroscience, Thomas Jefferson University, 901 Walnut St. Suite 400, Philadelphia, PA 19107, USA. ${ }^{11}$ Department of Neurosurgery, Thomas Jefferson University, Philadelphia, PA 19107, USA. ${ }^{12}$ Penn Image Computing \& Science Lab, University of Pennsylvania, Philadelphia, PA 19143, USA. ${ }^{\varpi}$ email: Michael.sperling@jefferson.edu
} 
EEG (SEEG) electrodes could similarly be used to reduce or obviate the need for prolonged epilepsy monitoring unit (EMU) evaluations to capture seizures.

ECoG can identify brain regions with high rates of inter-ictal epileptiform spikes ${ }^{1}$ and help localize the epileptogenic zone (EZ), which is the hypothetical region necessary and sufficient for seizure generation ${ }^{8}$. Results from several studies suggest high-frequency oscillations (HFOs: ripples $80-250 \mathrm{~Hz}$, fast ripples $250-600 \mathrm{~Hz}$ ) ${ }^{3,4,6,7,9-11}$ is a more specific biomarker of EZ than interictal spikes. Ripples occur more frequently than fast ripples but are thought to be less specific, because they are more often generated by physiological mechanisms ${ }^{9,10}$. Several groups have reported that unresected fast ripples in intraoperative ECoG recordings predict poor post-operative seizure outcome $e^{3,4,6,7,11-15}$. When spikes and HFOs coincide, or ripples and fast ripples coincide ${ }^{15}$, the biomarker is thought to be even more specific for $\mathrm{EZ}^{3,16-18}$.

Few studies have compared the spatial accuracy of spikes and HFOs in intraoperative ECoG recordings to extraoperative prolonged recordings for identifying the $\mathrm{EZ}^{19-23}$. Studies have found anesthesia and analgesia can affect the rate of these biomarkers ${ }^{24}$, and also the spatial distribution with respect to the $\mathrm{EZ}^{23}$. A recent metaanalysis $^{22}$ suggests that these studies report conflicting results. Whether anesthesia affects the morphology of HFOs is also unknown. Since electrocorticography is commonly performed, and HFOs may replace or supplement spikes in tailoring resection during $\mathrm{ECoG}^{25}$, the accuracy of spikes and HFOs for the EZ in intraoperative recordings must be further clarified with respects to the influence of anesthesia and analgesia.

Prolonged recordings using subdural or SEEG electrodes are advantageous compared to intraoperative recordings, because they are much more likely to capture spontaneous seizures that specify the seizure onset zone (SOZ). The EZ may not always encompass all of the SOZ due to both volume conduction and a possible ictal core region ${ }^{26,27}$, and conversely localizing the SOZ may not identify the complete EZ because of limited temporal sampling during the EMU evaluation. Evidence suggests that HFOs recorded from prolonged implants during non-rapid eye movement (non-REM) sleep may identify the EZ better than the $\mathrm{SOZ}^{28,29}$. One contentious issue is whether the spatial distribution of HFO rates during non-REM sleep is stable ${ }^{15,30,31}$. One recent large study found that the classification accuracy of fast ripples for the EZ was higher when a full night of non-REM sleep was analyzed as opposed to 5 -min segments ${ }^{32}$. This issue is of particular importance to comparing intraoperative to sleep recordings, since the duration of the intraoperative recordings are intrinsically limited.

In this study, we used intraoperative and extra-operative non-REM sleep recordings from SEEG electrodes, to determine whether the morphology of HFOs is altered by anesthesia. We also compared the rates of HFOs and sharp-spikes (i.e. spikes that when digitally filtered have HFO frequency content $80-600 \mathrm{~Hz}$, but do not have a distinct HFO on time frequency analysis) $)^{3,33,34}$, recorded in the two conditions, with each other and the location of both the clinically defined SOZ and the EZ. The EZ was defined as those electrode contacts with the highest rates of events, and then assessing whether removal of these electrodes or EZ was predictive of the post-operative seizure outcome. Our goal was to determine whether intraoperatively recorded HFOs using SEEG electrodes could obviate the need for prolonged iEEG.

\section{Results}

Patient characteristics, anesthesia, and analgesia. A total of 16 patients with medically refractory epilepsy were included in this study, 11 of them were male (Table S1). The patients had diverse etiologies of their epilepsy (Table S1) with 25\% who had non-lesional epilepsy on MRI. Four patients had mesial-temporal lobe epilepsy (MTLE); 2 patients had lateral temporal lobe epilepsy; 4 patients had temporal lobe epilepsy plus other region(s); and 6 patients had extra-temporal lobe epilepsy (Table S1). In the operating room, 2 of the patients were anesthetized with $\sim 1 \%$ exhaled sevoflurane, 12 patients with $\sim 2 \%$ exhaled sevoflurane, and 2 patients with propofol $90 \mathrm{mcg} / \mathrm{kg} / \mathrm{min}$. As analgesia 7 patients received a continuous infusion of $\sim 0.8 \mathrm{ug} / \mathrm{kg} / \mathrm{h}$ remifentanil, 3 patients received $\sim 1.6 \mathrm{ug} / \mathrm{kg} / \mathrm{h}$ remifentanil, 1 patient received $0.05 \mathrm{ug} / \mathrm{kg} / \mathrm{h}$ sufentanil. Five patients did not receive analgesia after induction (Table $\mathrm{S} 1$ ).

Differences in HFO power and frequency during intraoperative recordings compared to non-REM sleep. Since we quantified the power and spectral frequency of each HFO event, we first asked if anesthesia effected these measures. We used a generalized linear mixed-effects model to account for inter-contact differences in the matched electrodes $(n=530)$. We found that HFO power was decreased in the intraoperative recordings relative to the non-REM recordings for all HFO types, except for $\mathrm{fRonO}(p<0.01$, Fig. 1 , Table 1$)$. We next asked if this difference was influenced by anesthesia type. For RonO, we found the largest relative differences in the mean power for sevoflurane $2 \%$ and propofol compared to sevoflurane $1 \%$ (Figure S1, Table S2, $p<0.05)$. However, this comparison was indirect such that anesthesia related differences in ripple power were not compared across the intraoperative condition. Furthermore, the two patients that received sevoflurane $1 \%$ had smaller RonO power during sleep than the others, which could be related to their poor outcome (Table S1).

$\mathrm{RonO}$ and RonS spectral frequency was slightly decreased in the intraoperative recordings relative to the sleep recordings, and fRonO and fRonS frequency was increased in the intraoperative recordings $(p<0.01$, Fig. 1 , Table 1). We also examined whether the SOZ influenced HFO power and frequency, and found a relatively weak but sometimes significant correlation (Table 1).

Effects of non-REM sleep and anesthesia dose and type on HFO rate in SOZ and non-SOZ. We next asked for each of the HFO subtypes and sharp-spikes: (1) whether rates differed in the intraoperative and non-REM sleep condition; (2) whether rates were increased in the SOZ; (3) if the intraoperative and non-REM sleep recordings differed in the magnitude or significance of this effect; and (4) whether anesthesia and analgesia selection were significant factors interacting with this effect. We found that rates of some of the HFO subtypes were comparatively increased during the non-REM sleep recordings compared to the intraoperative record- 

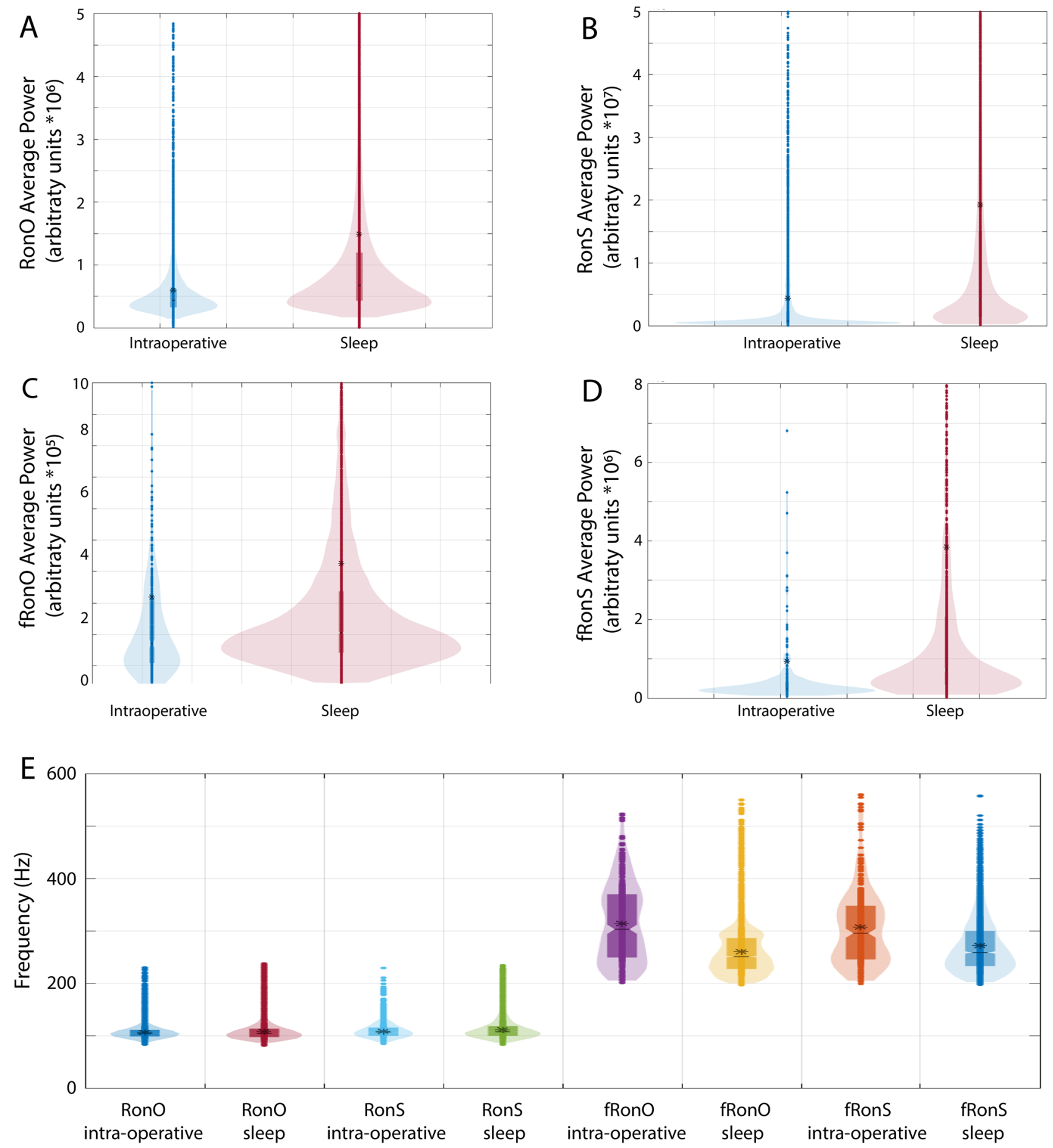

Figure 1. Differences in HFO average power and frequency by recording condition (intraoperative vs. non-REM sleep). Violin plots of ripples on oscillations (RonO, A), ripples on spikes (RonS, B), fast ripples on oscillations (fRonO, C), and fast ripples on spikes (fRonS, D) average power. HFO power was greater for all the event types, except fRonO, during non-REM sleep (red) than intraoperative recording (blue, $p<0.01$ ). (E) Violin plots of HFO spectral frequency. There was a small, but significant, increase in RonO and RonS frequency $(p<0.01)$ and decrease in $\mathrm{fRonO}$ and fRonS frequency $(p<0.01)$ during non-REM sleep than during intraoperative recording. Asterisk indicates mean.

ings in the matched contacts (repeated measures two-way ANOVA RonO: $\mathrm{F}=113.9, p<0.001$; $\mathrm{fRonO}$ : $\mathrm{F}=36.15$, $p<0.001$; fRonS: $\mathrm{F}=26.34, p<0.001$, Fig. 2A, Figure S2 d.f. $=524,1,2,1,2)$. However, the rate of RonS $(\mathrm{F}=6.79$, $p<0.01)$ and sharp-spikes $(\mathrm{F}=12.68, p<0.001)$ was higher in the intraoperative recording than during the sleep recording. The rate of HFOs and sharp-spikes was higher in the SOZ than in the non-SOZ during the combined 


\begin{tabular}{|c|c|c|c|c|c|c|c|}
\hline $\begin{array}{l}\text { Response } \\
\text { variable }\end{array}$ & $\begin{array}{l}\text { Intercept } \\
\text { estimate }\end{array}$ & $\begin{array}{l}\text { Intercept } p \\
\text { value }\end{array}$ & $\begin{array}{l}\text { Condition } \\
\text { estimate }\end{array}$ & $\begin{array}{l}\text { Condition } p \\
\text { value }\end{array}$ & SOZ estimate & SOZ $p$ value & d.f \\
\hline RonO Power & \begin{tabular}{|l|}
12.287 \\
{$[12.2-12.4]$} \\
\end{tabular} & $<1 \mathrm{e}-4$ & $1.291[1.14-1.3]$ & $<1 \mathrm{e}-4$ & $0.263[0.08-0.4]$ & $<0.005$ & 196,470 \\
\hline RonO Freq & $4.734[4.71-4.75]$ & $<1 \mathrm{e}-4$ & $\begin{array}{l}0.03[0.027- \\
0.032]\end{array}$ & $<1 \mathrm{e}-4$ & $\begin{array}{l}-0.025[-0.05 \\
\text { to } 0]\end{array}$ & 0.08 & 196,470 \\
\hline RonS Power & $\begin{array}{l}14.912 \\
{[14.6-15.2]} \\
\end{array}$ & $<1 \mathrm{e}-4$ & $0.97[0.77-1.17]$ & $<1 \mathrm{e}-4$ & $\begin{array}{l}-0.101[-0.46 \\
\text { to } .26]\end{array}$ & 0.58 & 31,479 \\
\hline RonS Freq & $4.678[4.66-4.68]$ & $<1 \mathrm{e}-4$ & $\begin{array}{l}0.022[0.016- \\
0.029]\end{array}$ & $<1 \mathrm{e}-4$ & $\begin{array}{l}0.003[-0.013 \\
\text { to } .02]\end{array}$ & 0.7 & 31,479 \\
\hline fRonO Power & $\begin{array}{l}12.251 \\
{[11.7-13.3]}\end{array}$ & $<1 \mathrm{e}-4$ & $0.3[-0.5$ to 1.11$]$ & 0.47 & $0.303[0.03-0.57]$ & 0.03 & 8416 \\
\hline fRonO Freq & $5.755[5.74-5.77]$ & $<1 \mathrm{e}-4$ & $\begin{array}{l}-0.1877[-0.2 \text { to } \\
-0.17]\end{array}$ & $<1 \mathrm{e}-4$ & $\begin{array}{l}-0.001[-0.02 \text { to } \\
-0.004]\end{array}$ & 0.003 & 8416 \\
\hline fRonS Power & \begin{tabular}{|l|}
13.602 \\
{$[12.6-14.5]$} \\
\end{tabular} & $<1 \mathrm{e}-4$ & $1.208[0.4-2.02]$ & 0.004 & $\begin{array}{l}-0.209[-1.0 \\
\text { to } .63]\end{array}$ & 0.62 & 2803 \\
\hline fRonS Freq & $5.706[5.68-5.72]$ & $<1 \mathrm{e}-4$ & $\begin{array}{l}-0.115[-0.13 \text { to } \\
-0.09]\end{array}$ & $<1 \mathrm{e}-4$ & $\begin{array}{l}0.029[0.013- \\
0.045]\end{array}$ & $<1 \mathrm{e}-4$ & 2803 \\
\hline
\end{tabular}

Table 1. Results of generalized linear mixed-effects models fitting HFO frequency and power. The randomeffect term was electrode, the fixed effects were recording condition (intraoperative vs. non-REM sleep), and SOZ. Brackets indicate $95 \%$ confidence interval.

sleep and intraoperative recordings (RonO: $\mathrm{F}=41.16, p<0.001$; RonS: $\mathrm{F}=9.97, p<0.005$; sharp-spike: $\mathrm{F}=13.81$, $p<0.001$; fRonO: $\mathrm{F}=7.0124, p<0.01$; fRonS: $\mathrm{F}=12.10, p<0.001$, Fig. $2 \mathrm{~A}$ ). The difference in rates was larger for all event during non-REM sleep than intraoperative recording (RonO:F=35.65, $p<0.001$; RonS: $\mathrm{F}=4.52$, $p<0.05$; sharp-spike: $\mathrm{F}=9.66, p<0.005$; fRonO: $\mathrm{F}=5.44, p<0.05$; fRonS: $\mathrm{F}=14.05, p<0.001$, Fig. $2 \mathrm{~A}$ ).

HFO generation has been shown to vary by neuroanatomic region ${ }^{35-39}$. We investigated if HFOs and sharpspikes rates varied by neuroanatomical lobe, and if this location interacted with the effect of the recording condition and the SOZ in the matched contacts. We found that the neuroanatomic location, by lobe, significantly influenced HFO and sharp-spike rates (Figure S3, Table S3). In contrast to other regions, higher ripple and sharp-spike rates, were seen in the frontal lobe, and to a less extent the parietal lobe, in the intraoperative recording relative to the sleep recording (Figure S3). Consequently, for all biomarker rates, except fRonO, the neuroanatomic location of the contact significantly interacted with the recording condition (Table S3). A weak but significant $(p<0.05)$ interaction was found between location and the SOZ for ripples and sharp-spikes only (Table S3). For all the biomarkers, the three-way interaction between SOZ, location, and condition was not significant. General anesthesia dose and type interacted with recording condition (intraoperative vs. non-REM sleep) to influence HFO rates for all HFO subtypes (RonO:F $=15.35, p<0.001$; RonS: $\mathrm{F}=8.43, p<0.001$ sharpspike: $\mathrm{F}=8.24, p<0.001$; fRonO: $\mathrm{F}=4.52, p<0.05$; fRonS: $\mathrm{F}=5.45, p<0.01$, Fig. $2 \mathrm{~B}$ ). For sharp-spikes, fRonO, and fRonS, but not RonO or RonS, there was also a three-way interaction $(p<0.05)$ between SOZ, anesthesia type, and the recording condition. However, we did not investigate this interaction further because of the small number of patients in this study.

The greatest decrease in HFO and spike rates was seen with propofol anesthesia, whereas higher concentrations of sevoflurane increased the rates of RonS and sharp-spikes (Fig. 2B). Visual analysis of the annotated iEEG revealed that $\sim 2 \%$ sevoflurane anesthesia often resulted in high amplitude sharply contoured rhythmic oscillations that were sometimes recognized as spikes by the detector (Figure S4), whereas propofol at a dose of $90 \mathrm{mcg} / \mathrm{kg} / \mathrm{min}$ did not produce a burst suppression pattern.

Analgesia infusion selection also significantly interacted with the condition of the recording for all HFO types and spikes, except RonO $(p<0.05)$. Paradoxically RonS and sharp-spike relative rates were higher in the patients who received no analgesia after induction, Figure S5. We could not investigate the interaction between choice of analgesia and anesthesia because the two were unmatched. There was no significant interaction between analgesia selection, condition, and the SOZ.

Classification of the SOZ using intraoperative and sleep HFO rates. Since HFO rates were increased in the SOZ in the intraoperative and non-REM sleep recordings, we next used receiver-operating characteristic (ROC) curve analysis to assess how well the rate of HFOs on each matched electrode contact $(n=530)$ during intraoperative and non-REM sleep recordings classified the SOZ. Using boot-strap analysis, we found that the area under the ROC (AUROC) curve was larger for RonO and RonS during non-REM sleep recordings than during the intraoperative recordings, and was significantly larger for sharp-spikes, fRonO, and fRonS ( $p<0.05, n=1000$ surrogates, Fig. 3A-E). RonO had the largest AUROC and was 0.72 [95\% confidence interval (CI) 0.66-0.77] for intraoperative recordings, and 0.80 [95\% CI 0.75-0.84] for sleep recordings. During the intraoperative and sleep recordings, the AUROC curve was significantly smaller for fRonO and fRonS than RonO $(p<0.05)$, but this may be due to a plateau effect at a limited specificity seen in the curves for fRonO and fRonS.

Since only a subset of the total number of electrodes were recorded in the intraoperative setting and in some cases, were outside the SOZ, we repeated the ROC curve analysis using rates of HFOs recorded from all electrodes 
$A_{1}$

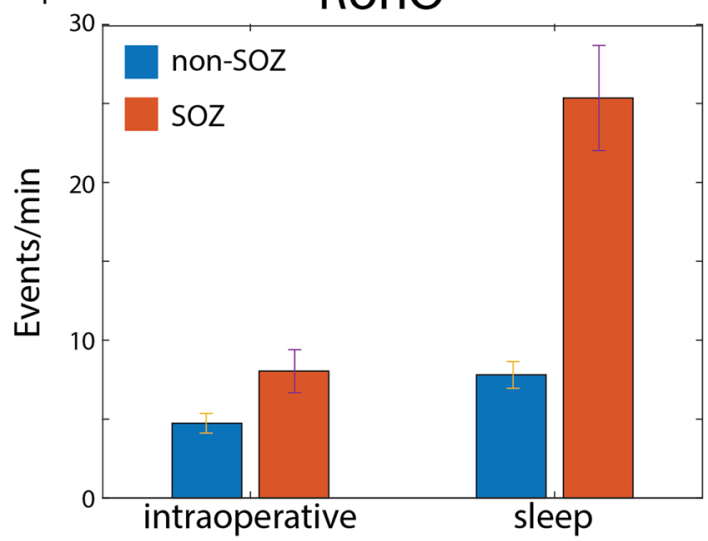

$\mathrm{A}_{3}$
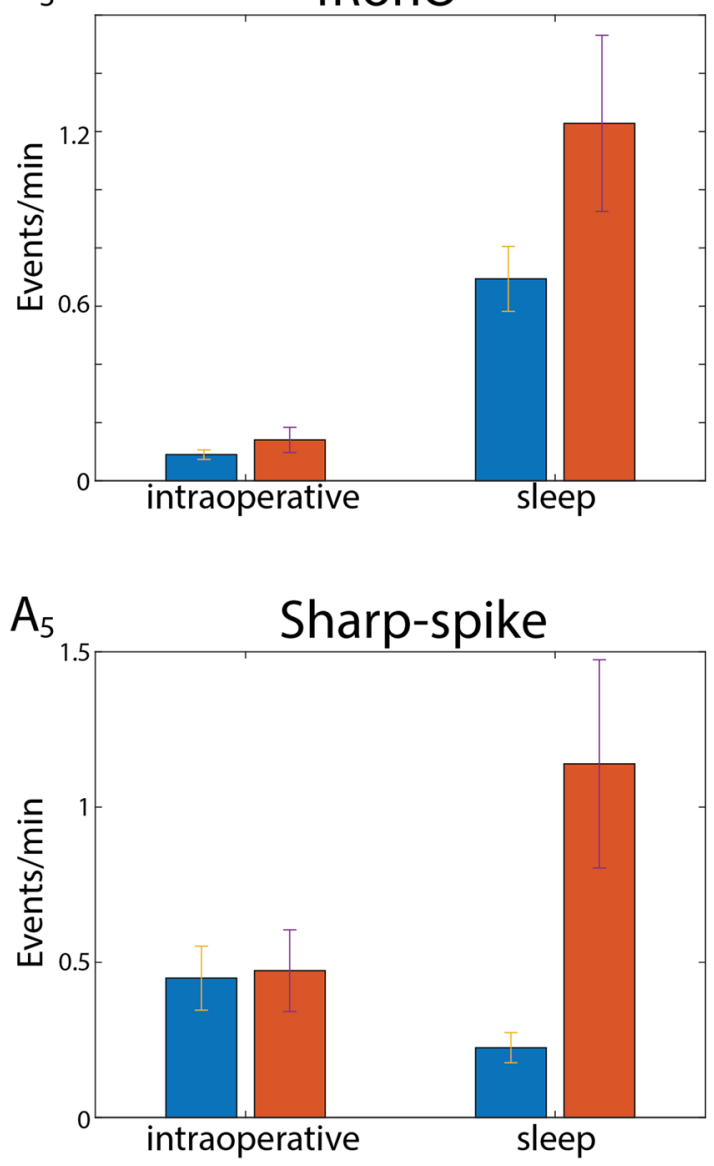

$\mathrm{A}_{2}$

RonS

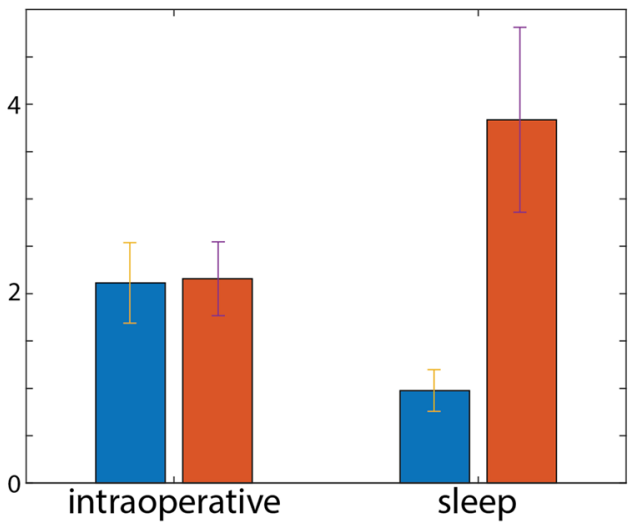

$\mathrm{A}_{4}$

fRons
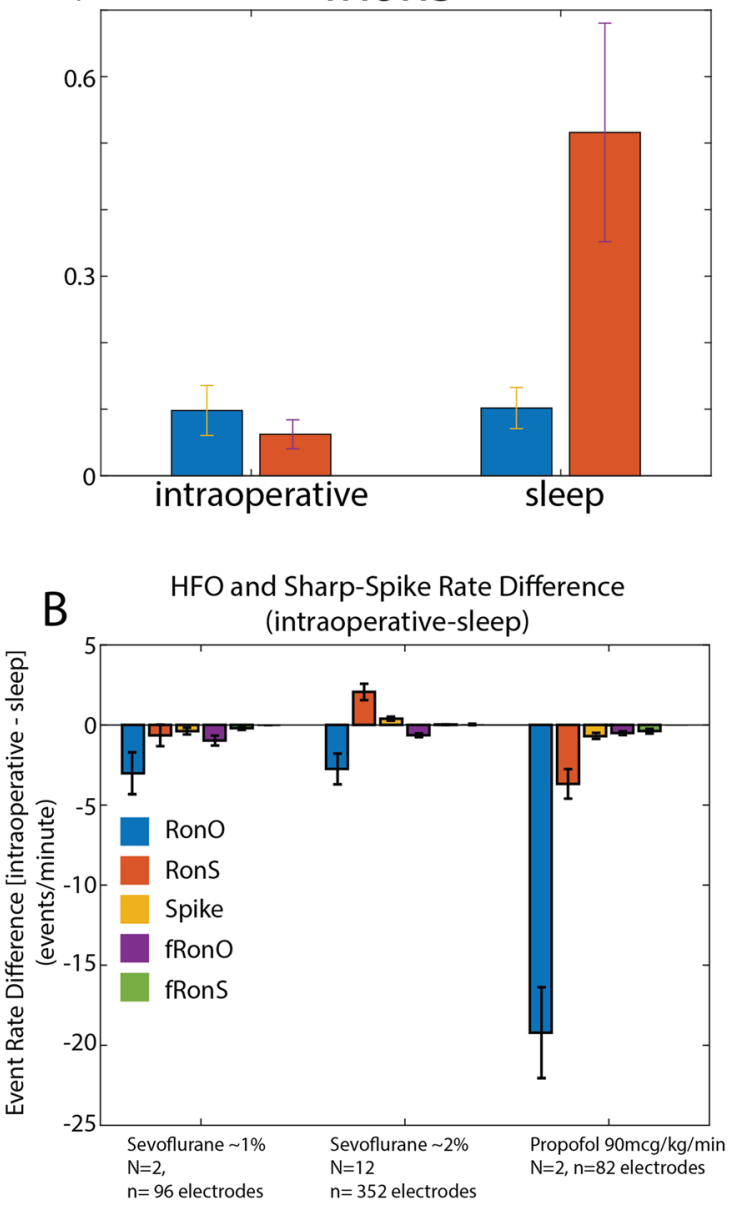

Figure 2. Mean HFO and spike rates in relation to the SOZ, recording condition, and anesthesia. (A1-5) A comparison of mean event rates in the non-SOZ (blue) and SOZ (orange) in the intraoperative (left) and sleep (right) condition measured from matched electrode contacts. The rate of RonO (A1), fRonO (A3), and fRonS (A4) was higher during sleep than intraoperative recording (rmANOVA, $p<0.05, \mathrm{~N}=16$ patients, d.f. $=523,1,1,2$ contacts). For both conditions combined, the rate was higher in the SOZ than the non-SOZ for all the individual biomarkers $(p<0.01)$. However, the difference in the rate of HFOs between SOZ and NSOZ was larger during sleep than intraoperative recordings $(\mathbf{A 1}-\mathbf{5}, p<0.05)$. (B) Differences in HFO and spike rates (intraoperative minus sleep) for each matched electrode contact with respect to anesthesia type and dose. In most cases, rates of HFO were lower during anesthesia than sleep, indicated by negative values in bar chart. Note RonS and spikes were higher during 2\% sevoflurane than non-REM sleep. Error bars indicate standard error of the mean (S.E.M). 
A

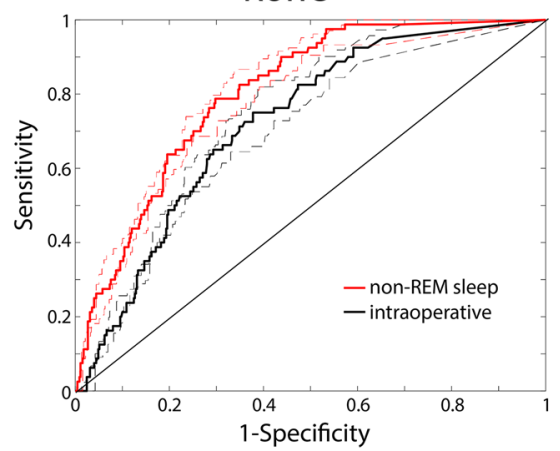

D

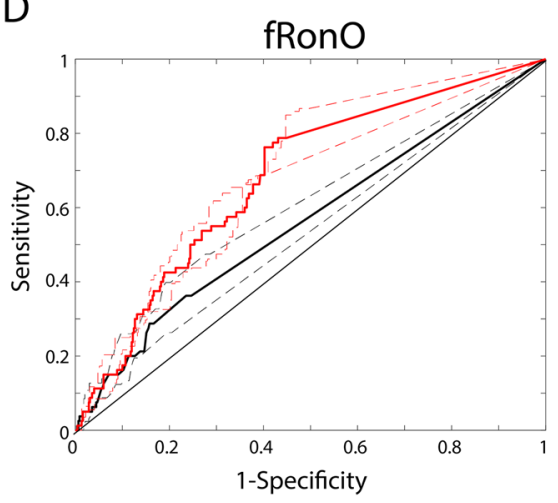

B

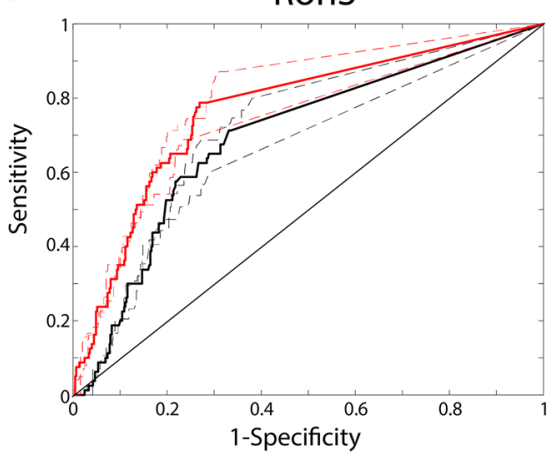

E

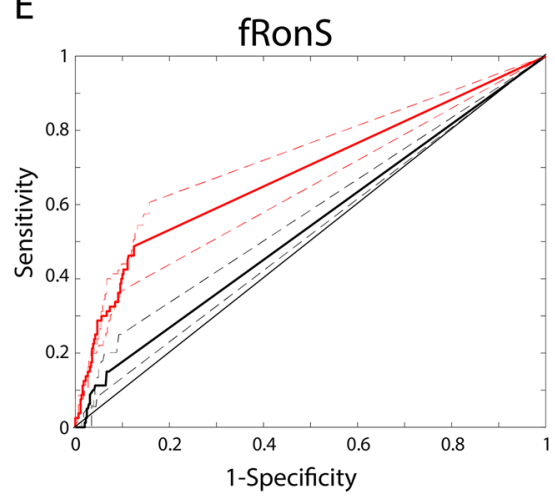

C

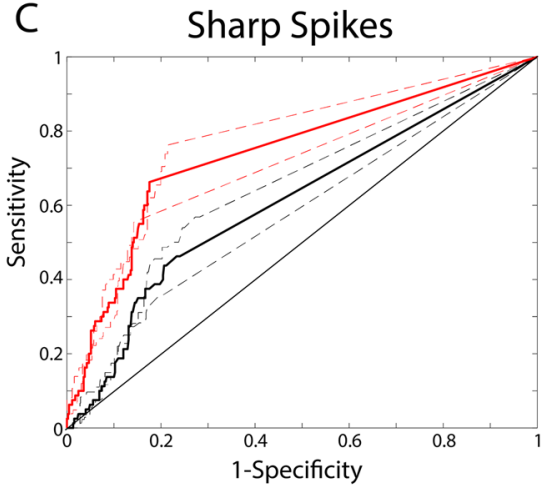

$\mathrm{F}_{1}$

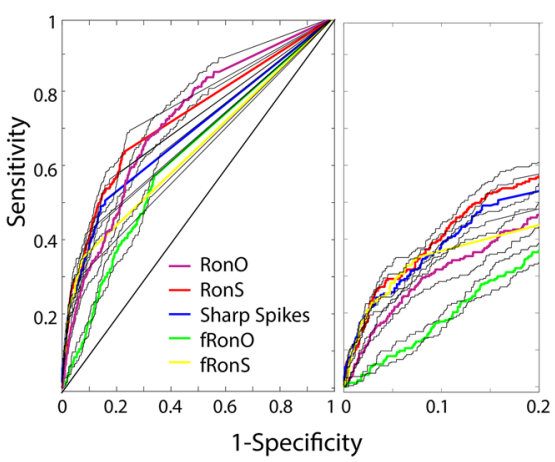

Figure 3. Comparison of receiver-operating characteristic (ROC) curves for different HFO subtype and sharp spike rates (A-E) in classifying the SOZ during intraoperative recordings ( $\mathrm{n}=530$ contacts, black) and the same matched electrode contacts during non-REM sleep recordings $(n=530$, red). Dashed lines indicate 95\% confidence intervals (CI) for the ROC curves. Only sharp-spikes and fRonS had a significantly larger area under the ROC curve (AUROC) in the sleep recordings $(p<0.05)$. (F) ROC curves for the HFO subtype and sharp spike rates using all contacts $(n=2630)$, including those unmatched, for classifying the SOZ sites during the non-REM sleep recordings (F1). At high specificities (F2), the partial AUROC of RonO and fRonO were inferior to sharp-spikes and HFOs on spikes $(p<0.5)$. Black lines indicate 95\% CI.

during non-REM sleep $(n=2630)$, including those that were unmatched. We found that at specificities $>80 \%$, RonO and fRonO were inferior to sharp-spikes and HFOs on spikes for classifying the SOZ (Fig. 3F, $p<0.05$ ).

Differences in the spatial distribution of HFOs and sharp-spikes in the intraoperative and non-REM sleep condition. To better understand the within-patient differences in HFO generation in the intraoperative and non-REM sleep recordings we calculated the scalar product for all pairs of HFO vectors during the two conditions using the paired contacts. We found that in some patients, HFOs and spikes were very reliable between the two conditions (Fig. $4 \mathrm{~A}, p<0.05, \mathrm{n}=2000$ surrogates). However, in other patients, the spatial distribution was less reliable or completely different (Fig. 4B, Figure S6). Overall, fRonO were the least reliable of all the event types across the 16 patients (Fig. $4 \mathrm{~A}$ ).

Comparison of resection of HFO and sharp-spike positive regions in individual patients with post-operative seizure outcome. To determine if HFOs and sharp-spikes recorded intraoperatively and during sleep define the EZ, we used an established method ${ }^{6,7,15}$ to define the contacts that exhibited the highest rate of events in each patient as the putative EZ. In eight patients, post-resection imaging was available, and we asked if the putative EZ was completely within the resection cavity. Four of the patients were not seizure free after surgery (Table S1). In two of the eight patients the resected region was not sampled by the intraoperative recording. In the intraoperative recordings, we found that the EZ defined by fRonO and fRonS trended the most sensitive for predicting failure. However, specificity and PPV were low (Table 2). Interestingly, these same events recorded from the same matched contacts during sleep performed slightly worse, but this difference was not statistically significant (Table 2). In the sleep recordings from all contacts, including those unmatched, fRonO trended the most sensitive and accurate among all the biomarkers, and also trended better than almost all of the biomarkers in the intraoperative recordings which had a limited montage (Table 2). However, even in the sleep recordings from all the electrodes, two seizure free patients had electrodes with fRonO that were outside the margins of resection. 
A

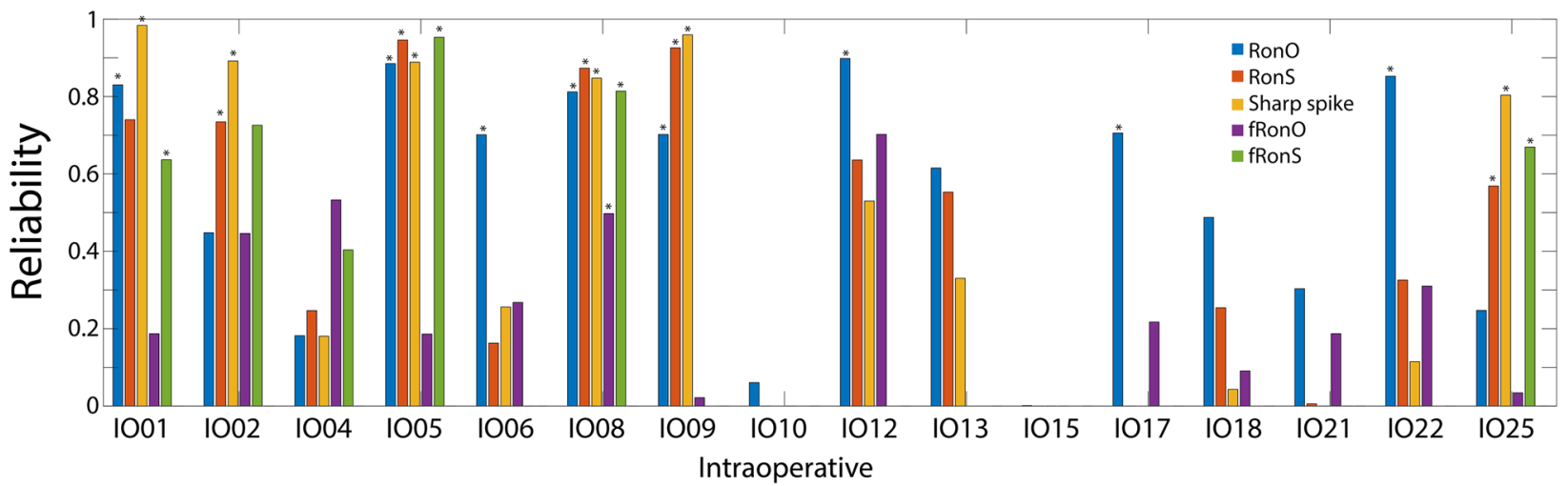

B

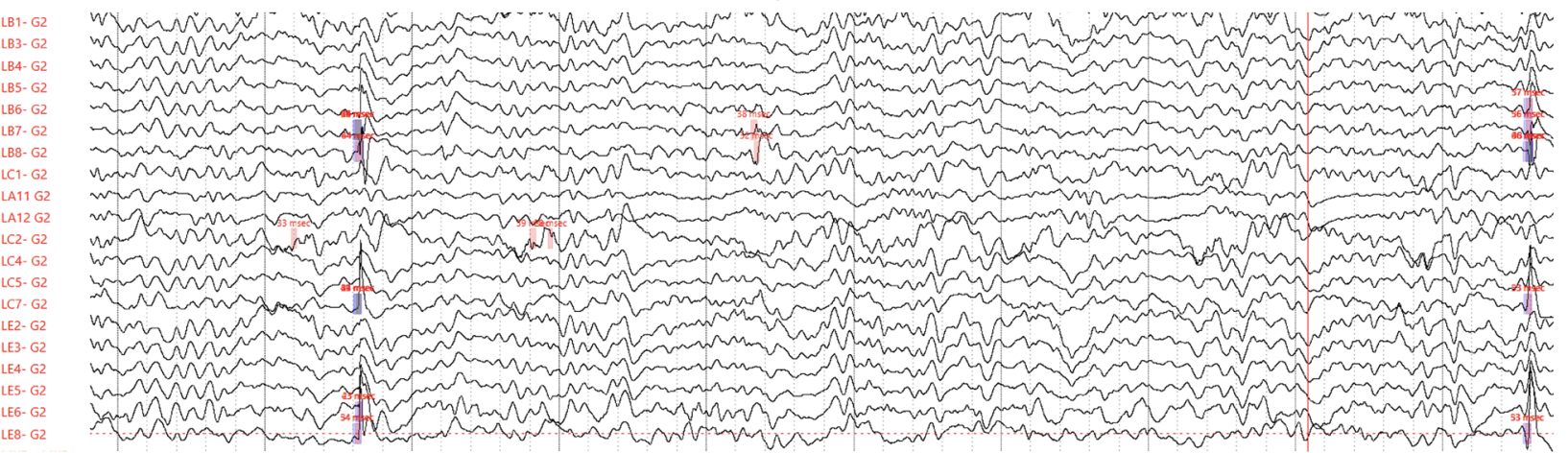

E8- G2

non-REM sleep

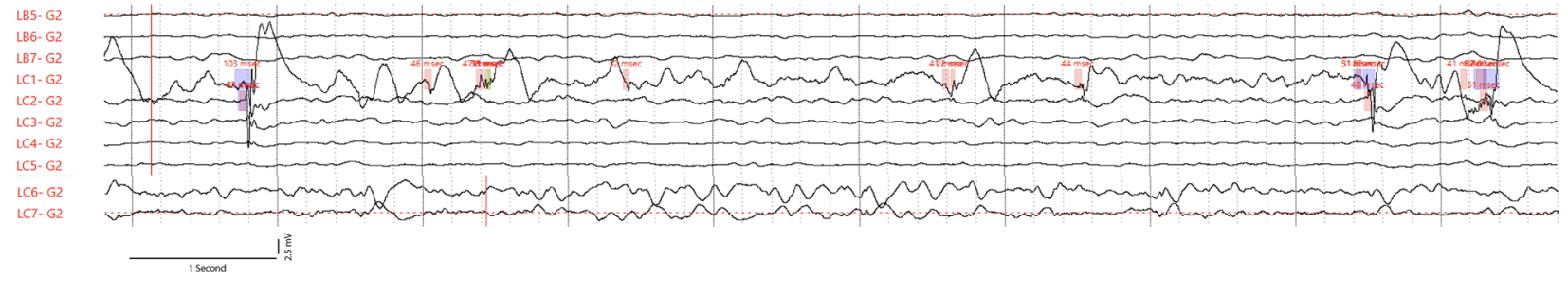

Figure 4. Evaluation of reliability in the spatial distribution of HFOs and sharp-spikes during the intraoperative and non-REM sleep recordings from matched electrode contacts. (A) The reliability measure computed from the scalar product for all pairs of HFO vectors between the two conditions for the 16 patients. Asterisk indicates significant reliability $(p<0.05)$ with respect to a random distribution $(n=2000$ surrogates $)$. (B) Intraoperative recording from patient IO015 shows RonS (pink and blue) in lateral temporal contacts (LB7,8,LC7), but during non-REM sleep recordings RonS, RonOs (pink), and fRonOs (green) appeared on in a mesial temporal contact (LC1), which was part of the SOZ.

\section{Discussion}

In summary, intraoperative recordings under anesthesia altered HFO morphology, rate, and in some patients, the spatial distribution compared to HFO from the same electrode contacts recorded during non-REM sleep. Overall, HFOs and sharp-spikes delineated the SOZ more accurately in the non-REM sleep than intraoperative recordings (Table 3). The neuroanatomic location of the SOZ did not significantly influence this effect. When the EZ was defined using all contacts during non-REM sleep, fRonO trended the best predicting post-operative seizure outcome, but the specificity and PPV were relatively low (Table 3). Thus, intraoperative SEEG recording is not a substitute for prolonged non-REM sleep recordings for identifying epileptogenic regions using interictal biomarkers.

HFOs exhibited decreased power under anesthesia, but the difference did not meet significance for fRonO. Also, ripples exhibited a slight but significant decrease in frequency, while fast ripples a large increase in frequency under anesthesia. Fast ripples are thought to be generated by clusters of pathologically interconnected neurons (PIN clusters) ${ }^{40}$ or out of phase firing of pyramidal neurons ${ }^{41}$. Ripples are generated by synchronized inhibitory post-synaptic potentials ${ }^{42}$. Propofol and isoflurane are both $\mathrm{GABA}_{\mathrm{A}}$ receptor agonists, but isoflurane also inhibits NMDA receptors ${ }^{43}$. Our results suggest that, during HFO generation, propofol and isoflurane decrease the number of recruited synchronized neurons. In the case of fast ripples, this theory is supported by the notion that event spectral frequency is inversely proportional to the size of the network ${ }^{44}$. More work is required to determine whether these changes alter the specificity of the biomarkers for epileptogenic regions. One possibility is, that in the intraoperative recordings, fast ripples may behave more like ripples generated during non-REM sleep because anesthesia increases the spectral frequency. 


\begin{tabular}{|l|l|l|l|l|l|}
\hline & Sensitivity & Specificity & PPV & NPV & Accuracy \\
\hline RonO intraop & $0.67[0.11-0.88]$ & $0.67[0.22-0.96]$ & $0.67[0.22-0.96]$ & $0.67[0.36-1.0]$ & $0.67[0.22-0.96]$ \\
\hline RonO sleep & $0.67[0.11-0.88]$ & $0.67[0.22-0.96]$ & $0.67[0.22-0.96]$ & $0.67[0.36-1.0]$ & $0.67[0.22-0.96]$ \\
\hline RonO all & $1.0[0.63-1.0]$ & $0.0[0.0-0.36]$ & $0.50[0.03-0.65]$ & $0.0[0.0-0.0]$ & $0.50[0.09-0.75]$ \\
\hline RonS intraop & $0.67[0.12-0.89]$ & $1.0[0.54-1.0]$ & $1.0[0.54-1.0]$ & $0.75[0.23-0.96]$ & $0.83[0.36-0.99]$ \\
\hline RonS sleep & $0.33[0.0-0.64]$ & $0.67[0.23-0.96]$ & $0.50[0.12-0.88]$ & $0.50[0.04-0.78]$ & $0.50[0.12-0.88]$ \\
\hline RonS all & $0.75[0.35-0.97]$ & $0.25[0.0-0.52]$ & $0.50[0.16-0.84]$ & $0.50[0.09-0.76]$ & $0.50[0.16-0.84]$ \\
\hline SSpike intraop & $0.67[0.36-1.0]$ & $0.67[0.36-1.0]$ & $0.67[0.11-0.88]$ & $0.67[0.36-1.0]$ & $0.67[0.0-0.64]$ \\
\hline SSpike sleep & $0.33[0.22-0.96]$ & $0.67[0.23-0.96]$ & $0.50[0.12-0.88]$ & $0.50[0.04-0.77]$ & $0.50[0.12-0.88]$ \\
\hline SSpike all & $0.75[0.35-0.97]$ & $0.25[0.0-0.52]$ & $0.50[0.16-0.84]$ & $0.50[0.09-0.76]$ & $0.50[0.16-0.84]$ \\
\hline fRonO intraop & $1.0[0.54-1.0]$ & $0.33[0.04-0.77]$ & $0.60[0.54-1.0]$ & $1.0[0.54-1.0]$ & $0.67[0.22-0.96]$ \\
\hline fRonO sleep & $0.67[0.54-1.0]$ & $0.33[0.04-0.78]$ & $0.50[0.0-0.64]$ & $0.50[0.0-0.64]$ & $0.5[0.12-0.88]$ \\
\hline fRonO all & $1.0[0.63-1.0]$ & $0.50[0.16-0.84]$ & $0.67[0.35-0.97]$ & $1.0[0.63-1.0]$ & $0.75[0.63-1.0]$ \\
\hline fRonS intraop & $1.0[0.54-1.0]$ & $0.33[0.0-0.64]$ & $0.60[0.04-0.77]$ & $1.0[0.54-1.0]$ & $0.67[0.12-0.88]$ \\
\hline fRonS sleep & $0.67[0.22-0.96]$ & $0.33[0.12-0.88]$ & $0.50[0.22-0.96]$ & $0.50[0.04-0.77]$ & $0.50[0.0-0.64]$ \\
\hline fRonS all & $0.75[0.25-0.92]$ & $0.25[0.09-0.76]$ & $0.50[0.35-0.97]$ & $0.50[0.09-0.76]$ & $0.50[0.09-0.76]$ \\
\hline
\end{tabular}

Table 2. Sensitivity, specificity, positive predictive value (PPV), negative predictive value (NPV, and accuracy of remaining HFO and sharp-spike (SSpike) positive regions (see "Methods" section) for predicting surgical failure (Engel Class $\geq 2$ ) in the intraoperative and electrode matched non-REM sleep recordings $(n=6)$, and the sleep recordings from all contacts $($ all, $\mathrm{n}=8)$. Brackets indicate $95 \%$ confidence intervals.

\begin{tabular}{|c|c|c|c|c|c|c|c|}
\hline Bio-marker & $\begin{array}{l}\text { Effect of anesthesia } \\
\text { on HFO power }\end{array}$ & $\begin{array}{l}\text { Effect of anesthesia } \\
\text { on HFO spectral } \\
\text { content }\end{array}$ & $\begin{array}{l}\text { Effect of anesthesia } \\
\text { (sevoflurane } 2 \% \text { ) on } \\
\text { event rate }\end{array}$ & $\begin{array}{l}\text { Overall accuracy } \\
\text { for SOZ (both } \\
\text { conditions) }\end{array}$ & $\begin{array}{l}\text { Sensitivity for } \mathrm{SOZ} \\
\text { at high specificities } \\
\text { (sleep) }\end{array}$ & $\begin{array}{l}\text { Effect of anesthesia } \\
\text { on SOZ accuracy }\end{array}$ & $\begin{array}{l}\text { EZ accuracy, (non- } \\
\text { REM sleep) }\end{array}$ \\
\hline RonO & - & - & - & $* * *$ & ** & $-1=$ & * \\
\hline RonS & - & - & + & $* *$ & $* * *$ & $-1=$ & * \\
\hline Sharp-spike & $\mathrm{n} / \mathrm{a}$ & $\mathrm{n} / \mathrm{a}$ & + & * & $* * *$ & - & * \\
\hline fRonO & $-1=$ & ++ & - & * & * & - & $* *$ \\
\hline fRonS & - & ++ & - & * & $* * *$ & - & * \\
\hline
\end{tabular}

Table 3. Summary of findings. Minus sign indicates negative change, plus sign indicates positive change, minus and equals sign indicates negative trend, but no significant change. Single asterisk indicates relatively low value, double asterisk medium value, and triple asterisk relatively high value.

HFOs except for RonS exhibited lower rates under anesthesia. Notably, RonS and sharp-spike rates were elevated in patients administered $2 \%$ isoflurane. Some studies have shown that higher concentrations of isoflurane increase the rate of spikes ${ }^{45}$, but in our study the increase may have been artifactual due to sharply contoured oscillations that would not have necessarily been marked as spikes on visual review. We did not redact these detections because of the ambiguity of the events, and the coinciding ripple events. Neuroanatomical differences may also have contributed, since RonS and sharp-spike rates were elevated during the intraoperative recording, relative to the sleep recording, in the frontal lobe and to a lesser degree the parietal lobe.

In comparing the anesthetics given in this study, propofol resulted in the largest decrease in HFO and spike rates in the absence of a burst-suppression pattern. A decrease in HFO rates with the use of propofol has been reported previously, and it is recommended to wean the propofol off before recording HFOs ${ }^{24}$. Opioid analgesia also decreased the rate of some HFO types and sharp-spikes. Typically, in electrocorticography recordings, these agents are given as boluses to increase spike rates ${ }^{46}$. In this study these agents were given as a constant infusion which has been shown in other studies to not increase spike rates ${ }^{47}$.

With respect to delineating the SOZ, HFO and sharp-spikes rates were relatively higher in the SOZ during non-REM sleep than during intraoperative recordings. Very few studies have compared the accuracy of intraoperative-recorded spikes and HFOs to localize the $\mathrm{SOZ}^{19,22-24}$. In one study ${ }^{23}$, however, HFOs recorded under propofol could define the SOZ, but accuracy between intra- and extra-operative recordings was not performed. Anesthesia could disrupt the normal expression of HFOs and spikes by epileptogenic tissue, while in contrast non-REM sleep facilitates the generation of spikes and HFOs in the SOZ and the EZ ${ }^{29,48}$. Frontal and parietal lobe seizures occur more frequently during non-REM sleep ${ }^{49,50}$, and pathological ripples there preferentially occur during the transition between the up-down state ${ }^{51-53}$. In other regions, such as the hippocampus ${ }^{10}$, the facilitation of pathological HFOs and spikes by the up-down state is less pronounced. To examine potential causes of the observed decrease in accuracy of HFOs for epileptogenic regions in the intraoperative condition, future experiments could examine changes to HFO phase amplitude coupling to slower oscillations under anesthesia. 
Among the 8 patients with post-operative MRI, fRonO had the highest accuracy for localizing the EZ, which is consistent with many past studies relating resection of HFOs with outcome $e^{4,6,7,15,32,54,55}$. By contrast, fRonO rates had the lowest accuracy of all the HFO types for identifying the SOZ in both intraoperative and sleep recordings. This inconsistency highlights the differences between the SOZ and the EZ, and also the dangers of using the SOZ as a metric by which to evaluate the utility of HFOs for surgical planning ${ }^{8}$. Also, HFOs on spikes and sharp-spike rates were more sensitive for the SOZ at high specificities, but notably these events trended less accurate for delineating that EZ. The study was underpowered to detect significant differences between HFOs on oscillations and HFOs on spikes for delineating the EZ. Several studies have suggested that HFOs on spikes are more accurate ${ }^{3,54,56}$, and are tightly correlated with increases in single unit firing rate ${ }^{57}$. Results are consistent with the hypothesis pathological HFOs, and specifically fRonO, are a electrophysiological biomarker for localizing the EZ in epilepsy surgery.

This study was also underpowered to detect significant differences between the intraoperative and sleep recording of HFO to localize the EZ. The intraoperative recordings trended more accurate than the matched contact sleep recordings, this result may be spurious since the SOZ was often only partially sampled by the limited montage of the intraoperative electrode contacts. The accuracy of fRonO in the sleep recordings using all the contacts trended better than the intraoperative recordings. Thus, fRonOs in sleep recordings, with adequate spatial sampling, may be more accurate than intraoperative recordings for defining the EZ. Another indication that supports this conclusion is that $\mathrm{fRonO}$ were the least spatially reliable biomarker between the two recording conditions.

In several patients during intraoperative recordings, high rates of, HFOs on spikes and sharp-spikes were found outside the SOZ. One possible explanation is that these spikes were injury potentials from electrode implantation that have been previously reported in studies of intraoperative depth electrode recordings ${ }^{58}$. However, in the hippocampus, these injury potentials were found to last only $1 \mathrm{~min}$ after implantation ${ }^{59}$. In the majority of patients, high rates of ectopic spikes were not present. This is consistent with a prior report that found that spike rates in intraoperative recordings from hippocampal depth electrodes correlated with hippocampal sclerosis ${ }^{2}$. Besides the possibility of injury potentials, another potential cause of the poor spatial reliability of HFOs and sharp-spikes was that the sleep recordings were often longer in duration than the intra-operative recordings. Poor temporal sampling should influence low-rate events such as fRonO and fRonS most, which is consistent with our results.

Results from this study were drawn from relatively few patients. For the GLMM, ANOVA, and ROC analysis we increased statistical power by making contact-matched comparisons. We could not use this approach to analyze the accuracy of HFOs and sharp-spikes for the EZ. Also, less than half of the patients in this study had resections followed by post-operative MRI. A larger study is required to prove that the accuracy of HFOs and sharp-spikes is inferior in the intraoperative condition, and would also help to resolve the effects of specific types and doses of anesthesia on delineating the SOZ and the EZ.

As noted previously only a subset of the total number of electrodes were recorded in the operating room and in some cases, were outside the SOZ and EZ. This could be a reason why fRonOs recorded from all electrodes during sleep more accurately defined the EZ than the fRonOs recorded from the subset of electrodes in the operating room. Future experiments may require recording from all SEEG electrodes, but this should be carefully planned since this would prolong the patient's exposure to anesthesia while connecting all the electrodes. Alternatively, anesthesia could be weaned while the electrodes are being connected which may also improve delineation of the SOZ and the EZ.

In summary, our results suggest that localization of the SOZ and likely the EZ is more accurate using HFOs recorded during sleep than during HFOs recorded in the operating room (Table 3). An appropriately powered, likely multi-centered, study is needed to verify these results. Anesthesia affects the morphology and rate of HFOs. The utility of intraoperative SEEG recordings could be improved by increasing recording duration, tapering patients off anesthesia, and perhaps utilizing opioid boluses. Prolonged SEEG recordings remain essential because they permit electroclinical correlation of the SOZ. However, for patients who cannot tolerate long-term monitoring or have circumscribed lesions on MRI, the utility of intraoperative SEEG deserves further exploration. A lack of spatial reliability, and the possibility of injury potentials remain a concern.

\section{Methods}

All patients underwent intracranial monitoring with depth electrodes between 2016 and 2018 at Thomas Jefferson University (TJU) for the purpose of localization of the SOZ (Table 1). Intraoperative recordings were obtained from the anesthetized patients 10-15 min following implantation of all SEEG electrodes. In the operating room, 10 -min recordings were obtained from 3 to 7 depth electrodes per patient. We connected only a subset of the total number of electrodes in the operating room so as not to prolong the patient's exposure to anesthesia. Depth electrodes selection was based on the results from the patient's previous scalp EEG study. One-two days after implantation, for each patient a 10-60 min iEEG recording from all the depth electrodes containing large amplitude, delta-frequency slow waves (i.e., non-REM sleep) was selected for analysis. Only iEEG that was at least $4 \mathrm{~h}$ of seizure-free, and was free of low levels of muscle contamination, and other artifacts, was selected.

All recordings were referenced to an iEEG electrode contact positioned in the white matter. Clinical iEEG recordings $(0.016-600 \mathrm{~Hz})$ were acquired, at a $2 \mathrm{kHz}$ sampling rate, from the depth electrode contacts using a Nihon-Kohden 256-channel JE-120 long-term monitoring system (Nihon-Kohden America, Foothill Ranch, CA, USA). The study was approved by the TJU institutional review boards (IRB Control \#16F.592), and patients gave informed consent prior to participating in this research. All research was in accord with the office for human research protections. 
The seizure onset zone was defined by the attending epileptologist for each patient and did not include areas of early propagation. The non-SOZ included all remaining contacts and was often separated from the SOZ by sub-centimeter distances (Table 1). In all the patients the SOZ was not completely sampled by the intraoperative recordings, in 4 patients, the $\mathrm{SOZ}$ was not sampled at all by the intraoperative recording contacts.

HFO detection. HFOs and sharp-spikes were detected in the intraoperative and sleep iEEG using previously published methods ${ }^{3,34,60}$ implemented in Matlab (Mathworks, Natick, MA, USA). In brief, the HFO detector reduced muscle and electrode artifacts in the iEEG recordings using a independent component analysis (ICA)-based algorithm ${ }^{60}$. After applying this ICA-based method, ripples and fast ripples were detected in the referential and bipolar montage iEEG recordings per contact by utilizing a Hilbert detector, in which a 1000th order symmetric finite impulse response (FIR) band-pass filter in the $(80-600 \mathrm{~Hz})$ for ripples and $(250-600 \mathrm{~Hz})$ for fast ripples was applied, and (ii) a Hilbert transform was applied to calculate the instantaneous amplitude of this time series according to the analytic signal $\mathrm{z}(\mathrm{t})$

$$
\mathrm{z}(\mathrm{t})=\mathrm{a}(\mathrm{t}) \mathrm{e} \wedge \mathrm{i} \phi(\mathrm{t})
$$

where $\mathrm{a}(\mathrm{t})$ is the instantaneous amplitude and $\varnothing(\mathrm{t})$ is the instantaneous phase of $\mathrm{z}(\mathrm{t})$. Following the Hilbert transform, the instantaneous HFO amplitude function $[\mathrm{a}(\mathrm{t})]$ was smoothed using moving window averaging, the smoothed instantaneous HFO amplitude function was normalized using the mean and standard deviation of the time series, and a custom statistical threshold defined by the skewness of the normalized time series was used to detect the onset and offset of discrete/potential events.

HFO-like events can arise due to Gibb's phenomenon, i.e., high-pass filtering sharp transients, including epileptiform spikes ${ }^{33}$. To distinguish authentic HFOs from authentic HFOs on EEG spikes or spurious HFO due to filter ringing, we used a custom algorithm that performed topographic analysis of time-frequency plots for each $\mathrm{HFO}^{34}$. The algorithm also measured the power, spectral content, and duration of each $\mathrm{HFO}$ and categorized the HFO as an HFO on oscillation or HFO on spike. Following automatic detection of HFO and sharp-spikes, false detections of clear muscle and mechanical artifact were deleted by visual review in Micromed Brainquick (Venice, Italy).In almost all the patients, less than 3-5\% of the detections were deleted ${ }^{3}$.

Neuroimaging. T1-pre-implant and post-resection MRIs were obtained for each patient. Post-implantation CT scans were then co-registered with the MRIs using Advanced Neuroimaging Tools (ANTs) ${ }^{61}$ with neuroradiologist supervision, using an in-house pipeline (https://github.com/pennmem/neurorad_pipeline). The position of each electrode contact was localized to the Desikan-Killiany atlas ${ }^{62}$. Identification of the electrode contacts in the resection cavity was performed manually in itk-SNAP (http://www.itksnap.org/pmwiki/pmwiki.php).

Statistics. HFO frequency and power values were fit with generalized linear mixed-effects models in Matlab with contact as the random-effects term, and condition and $\mathrm{SOZ}$ as fixed-effects predictors. A comparison of HFO and sharp spike rates, by electrode contact, was carried out in Matlab using the RANOVA function with factors including SOZ, anesthesia or analgesia or neuroanatomic lobe of the electrode contact, and two levels for the recording condition. Receiver operating curves were generated using the perfcurve function in Matlab, and $95 \%$ confidence intervals were estimated using 1000 boot-strap replicas. We quantified the reliability of the distribution of HFO rates between the intraoperative recording and extraoperative recording. For each interval pair we computed the normalized scalar product of the spatial distribution of the HFO rates. To test the magnitude of the true scalar product against chance, we permuted $(\mathrm{N}=2000)$ the order of channels for the intraoperative and sleep conditions ${ }^{15}$. For the outcome analysis, HFO and sharp spike positive regions were defined as the channels whose rates exceeded the 95th percentile of the distribution of all contacts during the recording (intra-op, matched sleep contacts, all sleep contacts). We defined as true positive a patient whose event area was not fully located in the margins of the resection and the post-operative seizure outcome was Engel IIa or worse. The positive predictive value was calculated as $\mathrm{PPV}=\mathrm{TP} /(\mathrm{TP}+\mathrm{FP})$, negative predictive value as $\mathrm{NPV}=\mathrm{TN} /$ $(\mathrm{TN}+\mathrm{FN})$, sensitivity $=\mathrm{TP} /(\mathrm{TP}+\mathrm{FN})$, specificity $=\mathrm{TN} /(\mathrm{TN}+\mathrm{FP})$, and accuracy $=(\mathrm{TP}+\mathrm{TN}) / \mathrm{N}$. Estimates of the 95\% confidence intervals (CI) used the binomial method.

\section{Data availability}

The datasets generated during and/or analysed during the current study are available from the corresponding author on reasonable request.

Received: 6 September 2021; Accepted: 19 October 2021

Published online: 01 November 2021

\section{References}

1. Keene, D. L., Whiting, S. \& Ventureyra, E. C. Electrocorticography. Epileptic Disord. Int. Epilepsy J. Videotape 2, 57-63 (2000).

2. Cascino, G. D., Sharbrough, F. W., Jack, C. R., Parisi, J. E. \& O’Brien, P. C. Acute depth electrode investigations in temporal lobe epilepsy: Correlation with magnetic-resonance-imaging-based volume studies and pathology. J. Epilepsy 5, 49-54 (1992).

3. Weiss, S. A. et al. Visually validated semi-automatic high-frequency oscillation detection aides the delineation of epileptogenic regions during intra-operative electrocorticography. Clin. Neurophysiol. 129, 2089-2098 (2018).

4. Wu, J. Y. et al. Removing interictal fast ripples on electrocorticography linked with seizure freedom in children. Neurology 75 , 1686-1694 (2010).

5. Gelinas, J. N., Battison, A. W., Smith, S., Connolly, M. B. \& Steinbok, P. Electrocorticography and seizure outcomes in children with lesional epilepsy. Child's Nerv. Syst. 27, 381-390 (2011). 
6. van't Klooster, M. A. et al. Residual fast ripples in the intraoperative corticogram predict epilepsy surgery outcome. Neurology 85, 1. https://doi.org/10.1212/WNL.0000000000001727 (2015).

7. van't Klooster, M. A. et al. Tailoring epilepsy surgery with fast ripples in the intraoperative electrocorticogram. Ann. Neurol. 81, 664-676 (2017).

8. Jehi, L. The epileptogenic zone: Concept and definition. Epilepsy Curr. 18, 12-16 (2018).

9. Engel, J., Bragin, A. \& Staba, R. Nonictal EEG biomarkers for diagnosis and treatment. Epilepsia Open 3, 120-126 (2018).

10. Weiss, S. A. et al. Ripples have distinct spectral properties and phase-amplitude coupling with slow waves, but indistinct unit firing, in human epileptogenic hippocampus. Front. Neurol. 11, 174 (2020).

11. Fedele, T. et al. Automatic detection of high frequency oscillations during epilepsy surgery predicts seizure outcome. Clin. Neurophysiol. 127, 3066-3074 (2016).

12. Burnos, S. et al. The morphology of high frequency oscillations (HFO) does not improve delineating the epileptogenic zone. Clin. Neurophysiol. 127, 2140-2148 (2016).

13. Hussain, S. A. et al. Intraoperative fast ripples independently predict postsurgical epilepsy outcome: Comparison with other electrocorticographic phenomena. Epilepsy Res. 135, 79-86 (2017).

14. Jacobs, J. et al. Removing high-frequency oscillations. Neurology 91, e1040-e1052 (2018).

15. Fedele, T. et al. Resection of high frequency oscillations predicts seizure outcome in the individual patient. Sci. Rep. 7, 13836 (2017).

16. Wang, S. et al. Ripple classification helps to localize the seizure-onset zone in neocortical epilepsy. Epilepsia 54, 370-376 (2013).

17. Weiss, S. A. et al. Ripples on spikes show increased phase-amplitude coupling in mesial temporal lobe epilepsy seizure-onset zones. Epilepsia 57, 1916-1930 (2016).

18. Kumar, U., Li, L., Bragin, A. \& Engel, J. Spike and wave discharges and fast ripples during posttraumatic epileptogenesis. Epilepsia https://doi.org/10.1111/epi.16958 (2021).

19. Asano, E. et al. Is intraoperative electrocorticography reliable in children with intractable neocortical epilepsy?. Epilepsia 45, 1091-1099 (2004)

20. Kurita, N. et al. The effects of sevoflurane and hyperventilation on electrocorticogram spike activity in patients with refractory epilepsy. Anesth. Analg. 101, 517-523 (2005).

21. Orihara, A. et al. Effects of sevoflurane anesthesia on intraoperative high-frequency oscillations in patients with temporal lobe epilepsy. Seizure 82, 44-49 (2020).

22. Bayram, A. K. et al. Effect of anesthesia on electrocorticography for localization of epileptic focus: Literature review and future directions. Epilepsy Behav. 118, 107902 (2021).

23. Inada, T. et al. Effects of a stable concentration of propofol on interictal high-frequency oscillations in drug-resistant epilepsy. Epileptic Disord. 23, 299-312 (2021).

24. Zijlmans, M. et al. Epileptic high-frequency oscillations in intraoperative electrocorticography: The effect of propofol. Epilepsia 53, 1799-1809 (2012).

25. van't Klooster, M. A. et al. High frequency oscillations in the intra-operative ECoG to guide epilepsy surgery ("The HFO Trial"): Study protocol for a randomized controlled trial. Trials 16, 422 (2015).

26. Weiss, S. A. et al. Ictal high frequency oscillations distinguish two types of seizure territories in humans. Brain 136, 3796-3808 (2013)

27. Weiss, S. A. et al. Seizure localization using ictal phase-locked high gamma. Neurology 84, 2320-2328 (2015).

28. Frauscher, B. et al. High-frequency oscillations: The state of clinical research. Epilepsia 58, 1316-1329 (2017)

29. Klimes, P. et al. NREM sleep is the state of vigilance that best identifies the epileptogenic zone in the interictal electroencephalogram. Epilepsia 60, 2404-2415 (2019).

30. Gliske, S. V. et al. Variability in the location of high frequency oscillations during prolonged intracranial EEG recordings. Nat. Commun. 9, 2155 (2018).

31. Fedele, T., Ramantani, G. \& Sarnthein, J. High frequency oscillations as markers of epileptogenic tissue-End of the party?. Clin. Neurophysiol. 130, 624-626 (2019).

32. Nevalainen, P. et al. Association of fast ripples on intracranial EEG and outcomes after epilepsy surgery. Neurology 95, e2235-e2245 (2020).

33. Bénar, C. G., Chauvière, L., Bartolomei, F. \& Wendling, F. Pitfalls of high-pass filtering for detecting epileptic oscillations: A technical note on "false" ripples. Clin. Neurophysiol. 121, 301-310 (2010).

34. Waldman, Z. J. et al. A method for the topographical identification and quantification of high frequency oscillations in intracranial electroencephalography recordings. Clin. Neurophysiol. 129, 308-318 (2018).

35. Alkawadri, R. et al. The spatial and signal characteristics of physiologic high frequency oscillations. Epilepsia 55, 1986-1995 (2014).

36. Frauscher, B. et al. High-frequency oscillations in the normal human brain. Ann. Neurol. 84, 374-385 (2018).

37. Guragain, H. et al. Spatial variation in high-frequency oscillation rates and amplitudes in intracranial EEG. Neurology 90, e639-e646 (2018).

38. Motoi, H. et al. Quantitative analysis of intracranial electrocorticography signals using the concept of statistical parametric mapping. Sci. Rep. 9, 17385 (2019).

39. Salami, P. et al. Seizure onset location shapes dynamics of initiation. Clin. Neurophysiol. 131, 1782-1797 (2020).

40. Bragin, A., Mody, I., Wilson, C. L. \& Engel, J. Local generation of fast ripples in epileptic brain. J. Neurosci. 22, 2012-2021 (2002).

41. Foffani, G., Uzcategui, Y. G., Gal, B. \& de la Prida, L. M. Reduced spike-timing reliability correlates with the emergence of fast ripples in the rat epileptic hippocampus. Neuron 55, 930-941 (2007).

42. Stark, E. et al. Pyramidal cell-interneuron interactions underlie hippocampal ripple oscillations. Neuron 83, 467-480 (2014).

43. Brohan, J. \& Goudra, B. G. The role of GABA receptor agonists in anesthesia and sedation. CNS Drugs 31, 845-856 (2017).

44. Brunel, N. \& Wang, X.-J. What determines the frequency of fast network oscillations with irregular neural discharges: I. Synaptic dynamics and excitation-inhibition balance. J. Neurophysiol. 90, 415-430 (2003).

45. Ito, B. M., Sato, S., Kufta, C. V. \& Tran, D. Effect of isoflurane and enflurane on the electrocorticogram of epileptic patients. Neurology 38, 924-924 (1988).

46. Wass, C. T. et al. The effects of remifentanil on epileptiform discharges during intraoperative electrocorticography in patients undergoing epilepsy surgery. Epilepsia 42, 1340-1344 (2001).

47. Herrick, I. A., Craen, R. A., Blume, W. T., Novick, T. \& Gelb, A. W. Sedative doses of remifentanil have minimal effect on ECoG spike activity during awake epilepsy surgery. J. Neurosurg. Anesth. 14, 55-58 (2002).

48. von Ellenrieder, N., Dubeau, F., Gotman, J. \& Frauscher, B. Physiological and pathological high-frequency oscillations have distinct sleep-homeostatic properties. Neuroimage Clin. 14, 566-573 (2017).

49. Herman, S. T., Walczak, T. S. \& Bazil, C. W. Distribution of partial seizures during the sleep-wake cycle. Neurology 56, 1453-1459 (2001).

50. Loddenkemper, T. et al. Circadian patterns of pediatric seizures. Neurology 76, 145-153 (2011).

51. Frauscher, B. et al. Facilitation of epileptic activity during sleep is mediated by high amplitude slow waves. Brain 138, 1629-1641 (2015).

52. Ellenrieder, N., Frauscher, B., Dubeau, F. \& Gotman, J. Interaction with slow waves during sleep improves discrimination of physiologic and pathologic high-frequency oscillations (80-500 Hz). Epilepsia 57, 869-878 (2016). 
53. Song, I. et al. Bimodal coupling of ripples and slower oscillations during sleep in patients with focal epilepsy. Epilepsia 58, 19721984 (2017).

54. Jacobs, J. et al. High-frequency electroencephalographic oscillations correlate with outcome of epilepsy surgery. Ann. Neurol. 67, 209-220 (2010).

55. Haegelen, C. et al. High-frequency oscillations, extent of surgical resection, and surgical outcome in drug-resistant focal epilepsy. Epilepsia 54, 848-857 (2013).

56. Jacobs, J., Kobayashi, K. \& Gotman, J. High-frequency changes during interictal spikes detected by time-frequency analysis. Clin. Neurophysiol. 122, 32-42 (2011).

57. Guth, T. A. et al. Interictal spikes with and without high-frequency oscillation have different single-neuron correlates. Brain https:// doi.org/10.1093/brain/awab288 (2021).

58. Engel, J., Driver, M. V. \& Falconer, M. A. Electrophysiological correlates of pathology and surgical results in temporal lobe epilepsy. Brain 98, 129-156 (1975).

59. Ulbert, I. et al. In vivo laminar electrophysiology co-registered with histology in the hippocampus of patients with temporal lobe epilepsy. Exp. Neurol. 187, 310-318 (2004).

60. Shimamoto, S. et al. Utilization of independent component analysis for accurate pathological ripple detection in intracranial EEG recordings recorded extra- and intra-operatively. Clin. Neurophysiol. 129, 296-307 (2018).

61. Avants, B. B., Epstein, C. L., Grossman, M. \& Gee, J. C. Symmetric diffeomorphic image registration with cross-correlation: Evaluating automated labeling of elderly and neurodegenerative brain. Med. Image Anal. 12, 26-41 (2008).

62. Desikan, R. S. et al. An automated labeling system for subdividing the human cerebral cortex on MRI scans into gyral based regions of interest. Neuroimage 31, 968-980 (2006).

\title{
Acknowledgements
}

The authors would like to thank Dale and Ted Wyeth for their technical contribution as well as Dr. Lin Li. This work was fully supported by NIH K23 NS094633 (SAW), a Junior Investigator Award from the American Epilepsy Society (SAW), R01 NS106958 (RS), and R01 NS033310 (JE).

\section{Author contributions}

S.A.W.: conceptualization, methodology, software, investigation, writing original draft, review and editing, visualization, supervision, funding acquisition. R.J.S.: conceptualization, investigation, writing, funding acquisition. A.S.: investigation. C.W.: investigation. D.R.: investigation, visualization, writing S.D.: software. Z.W.: software, investigation, visualization. I.O.: investigation, writing. G.W.: conceptualization, writing, funding acquisition. J.E.: conceptualization, investigation, writing, funding acquisition. M.S.: conceptualization, methodology, software, investigation, writing, visualization, supervision, funding acquisition.

\section{Competing interests}

MS has served as Consultant/advisor: Medtronic, Neurelis; Speaker: International Medical Press, Medscape, Projects in Knowledge, Eisai, UCB Pharma Research support: Cavion, Cerevel, Eisai, Engage, Medtronic, Neurelis, SK Life Science, Takeda, UCB Pharma, Xenon. The other authors do not declare competing interests.

Additional information

Supplementary Information The online version contains supplementary material available at https://doi.org/ 10.1038/s41598-021-00894-3.

Correspondence and requests for materials should be addressed to M.R.S.

Reprints and permissions information is available at www.nature.com/reprints.

Publisher's note Springer Nature remains neutral with regard to jurisdictional claims in published maps and institutional affiliations.

\begin{abstract}
Open Access This article is licensed under a Creative Commons Attribution 4.0 International License, which permits use, sharing, adaptation, distribution and reproduction in any medium or format, as long as you give appropriate credit to the original author(s) and the source, provide a link to the Creative Commons licence, and indicate if changes were made. The images or other third party material in this article are included in the article's Creative Commons licence, unless indicated otherwise in a credit line to the material. If material is not included in the article's Creative Commons licence and your intended use is not permitted by statutory regulation or exceeds the permitted use, you will need to obtain permission directly from the copyright holder. To view a copy of this licence, visit http://creativecommons.org/licenses/by/4.0/.
\end{abstract}

(c) The Author(s) 2021 